\title{
Spin Relaxation by Transient Monopolar and Bipolar Optical Orientation
}

\author{
B. N. Murdin, K. Litvinenko, and D. G. Clarke \\ Advanced Technology Institute, University of Surrey, Guildford GU2 7XH, United Kingdom \\ C. R. Pidgeon and P. Murzyn* \\ Department of Physics, Heriot-Watt University, Edinburgh EH14 4AS, United Kingdom \\ P. J. Phillips, D. Carder, G. Berden, B. Redlich, and A. F. G. van der Meer \\ FOM Institute "Rijnhuizen", Post Box 1204, NL-3430BE Niewegein, The Netherlands \\ S. Clowes, J. J. Harris, and L. F. Cohen \\ Blackett Laboratory, Imperial College London, Prince Consort Road SW7 2BZ, United Kingdom
}

T. Ashley and L. Buckle

QinetiQ Ltd, St. Andrews Road, Malvern WR14 3PS, United Kingdom

(Received 9 December 2004; published 10 March 2006)

\begin{abstract}
We have used two-color time-resolved spectroscopy to measure the relaxation of electron spin polarizations in a bulk semiconductor. The circularly polarized pump beam induces a polarization either by direct excitation from the valence band, or by free-carrier (Drude) absorption when tuned to an energy below the band gap. We find that the spin relaxation time, measured with picosecond time resolution by resonant induced Faraday rotation in both cases, increases in the presence of photogenerated holes. In the case of the material chosen, $n$-InSb, the increase was from 14 to 38 ps.
\end{abstract}

PACS numbers: 72.25.Rb, 72.25.Fe, 78.47.+p

Spin polarizations have become a focus of interest in semiconductor physics in recent years, not least for their potential application in "spintronic" devices [1]. It has been shown that spin polarized electrons (or holes) can be injected from magnetic semiconductor materials into semiconductors $[2,3]$, that they can be coherently transported through a device [4], and that they can be controlled (or modulated) with an external electric field [5]. It remains challenging to improve these breakthroughs to the extent that they can be combined in a practical device [1]. A sufficiently long spin lifetime $\tau_{\mathrm{s}}$ is important in the design of structures which confine and/or transfer spin. It is therefore necessary to understand the establishment of a polarized spin population and subsequent spin relaxation mechanisms in both bulk and low dimensional semiconductors. A large amount of literature has been established to determine spin lifetimes by one or another form of timeresolved optical orientation techniques based on spin injection by pulsed interband pumping with circularly polarized light [4-13]. In this Letter we investigate and contrast the spin relaxation lifetimes as measured in a bipolar (interband) experiment with spin lifetimes measured in a monopolar regime. In the former, significant numbers of excess electrons and holes are photopumped, whereas most device proposals involve electrical injection and manipulation of one type of charge carrier only, ideally at room temperature and often zero magnetic field. To this end we compare in such conditions the results of interband versus intraband pumping of spins with picosecond pulses, in the same sample. We find that the difference is a factor of between 2 to 3 .

Only one previous experiment measured the lifetime of spins pumped with monopolar excitation in zero magnetic field. In that case hole lifetimes in $p$-type GaAs/AlGaAs quantum wells were measured at low temperature by steady state bleaching of the circularly polarized photocurrent response [14]. The spin lifetime estimated from the saturation intensity was found to be $\sim 20 \mathrm{ps}$ at liquid helium temperature. Our picosecond time-resolved measurements do not rely on theoretically derived absorption cross sections, were made on material at room temperature, and directly compare the bipolar and monopolar spin excitation and relaxation in the same sample. Our results show, counterintuitively, that photoholes slow down the electron spin relaxation in $n$-type materials (by a factor of 2 to 3 in our case).

We chose the narrow gap semiconductor (NGS) InSb for the present study because of the relative strength of the Drude absorption (due to the small effective mass) at wavelengths relatively near the interband absorption (associated with the small energy gap). This was done so that a single tuneable pump laser, in our case the free electron laser FELIX, could easily reach both regimes. NGSs may be important in future spintronics applications because of their high Rashba effect [15-17], $g$ factor, and mobility, etc. Indeed electrical observation of injection with long spin mean free path has been reported [18]. In spite of their potential, many fewer time-resolved optical measurements 
of spin relaxation in narrow gap materials have been described than in the GaAs system, because of the relative rarity of suitable long wavelength short pulse light sources. However, we and others have reported such measurement of lifetimes in $\mathrm{HgCdTe}$ [9], InSb [9], InAs [10-12], and InAs/GaSb superlattices [13]. For the same reason, to our knowledge, there have not been any reports of timeresolved monopolar excitation.

In bulk semiconductors three main spin relaxation processes have been found to be important in optical orientation experiments: the D'yakonov-Perel (DP) [19], the Elliott-Yafet (EY) [20], and the Bir-Aronov-Pikus (BAP) [21] mechanisms. The BAP mechanism is based on the electron-hole exchange interaction and its rate increases with the concentration of holes. The EY mechanism results from the fact that the eigenstates are mixed spin and so can be connected by interactions with phonons, etc. The rate $1 / \tau_{\mathrm{s}}^{\mathrm{EY}}$ is proportional to $1 / \tau_{p}$ where $\tau_{p}$ is the orbital momentum scattering time, which would again be increased by the presence of holes.

The DP mechanism results from the lack of inversion symmetry so that the spin-orbit interaction not only mixes the spins as described above but also lifts the spin degeneracy, even in the absence of an externally applied magnetic field. For small $\mathbf{k}$ in bulk crystals this splitting can be written as $\Delta E=\gamma k^{3}$, where $\gamma$ is the Dresselhaus spinsplitting coefficient. The splitting is equivalent to that which would be caused by an effective magnetic field resulting in the electron spin precessing at a rate determined by the splitting. Because of the mixing, injection of an electron with definite spin by circularly polarized light (or from a magnetic material) is not in a stationary state but a linear combination of the eigenstates. This superposition precesses coherently at a rate given by the spin splitting, which depends on $\mathbf{k}$. Furthermore, the direction of the precession vector $\boldsymbol{\Omega}$ also depends on $\mathbf{k}$. In an ensemble of electrons all having different $\mathbf{k}$ and hence precessing about different directions and at different rates, the macroscopic polarization unphases at a rate determined by the magnitude of $\Omega$. Linear momentum scattering changes the DP picture from the "ballistic" regime to the "diffusive" regime, and at elevated temperature it causes $\mathbf{k}$ and hence also $\boldsymbol{\Omega}$ for each electron to perform a random walk, slowing the DP unphasing, so that its rate $1 / \tau_{\mathrm{s}}$ is proportional to $\tau_{p}$. This is the so-called "motional narrowing," which occurs when $\Omega \tau_{p} \ll 1$. The presence of holes would be expected to increase $1 / \tau_{p}$ for the electrons, which would have the effect of increasing the electron spin lifetime $\tau_{\mathrm{s}}$, in contrast with the other two mechanisms (and we show below that this is indeed the case).

The diffusive DP relaxation rate is given by [19]

$$
\frac{1}{\tau_{\mathrm{DP}}}=\Omega^{2} \tau_{p}=\frac{Q \alpha^{2} E_{k}^{3}}{\hbar^{2} E_{G}} \tau_{p}
$$

where $E_{G}$ is the band gap, $E_{k}$ is the electron kinetic energy, and $\alpha$ is a measure of the spin splitting. The dimensionless parameter $Q$ depends on the electron distribution and scattering process, and is predicted to be 0.05 for impurity scattering and degenerate statistics [7]. Clearly the lifetime increases as the temperature (and hence also $E_{k}$ ) is reduced as is shown in the inset of Fig. 1.

At room temperature and zero magnetic field spin lifetimes of the order of $1 \mathrm{~ns}$ were reported for GaAs [22] (though of the order of hundreds of ns for low temperature), and $10-20$ ps for lightly $n$-type InSb and InAs [912], and have been interpreted with the DP mechanism. The expected difference in the DP lifetime between InSb and GaAs may be roughly estimated from band structure parameters at the band edge, since at the band edge where the spin splitting is cubic in $k, \alpha=\eta m_{e} / \sqrt{3-\eta}$, where $\eta=\Delta /\left(E_{G}+\Delta\right), m_{e}$ is the electron effective mass ratio and $\Delta$ is the spin orbit split-off energy [19]. Hence $\alpha=$ 0.045 for InSb and 0.027 for GaAs, so $\tau_{\mathrm{DP}} \propto 1 / \Omega^{2} \propto$ $E_{G} / \alpha^{2}$ is about 20 times smaller in InSb than in GaAs. Alternatively, using values of $\gamma=\alpha / \sqrt{8 E_{G} m_{e}^{3}}$ (atomic units) from [23] we find smaller absolute magnitude $\left(\alpha^{\mathrm{InSb}}=0.017\right)$, but larger ratio $\tau_{\mathrm{DP}}^{\mathrm{GaAs}} / \tau_{\mathrm{DP}}^{\mathrm{InSb}} \sim 80$ using $\tau_{\mathrm{DP}} \propto 1 / \gamma^{2}$.

The sample used for our study, ME1722, was $5 \mu \mathrm{m}$ thick InSb, grown by molecular-beam epitaxy technique on a semi-insulating GaAs substrate. It was $n$-type doped in order to have significant intraband free-carrier absorption, and the room temperature electron concentration and mobility measured with the Hall effect were $8 \times 10^{16} \mathrm{~cm}^{-3}$ and $54000 \mathrm{~cm}^{2} \mathrm{~V}^{-1} \mathrm{~s}^{-1}$, respectively. For reference we have also made measurements on a lightly doped sample, ME1654 $\left(1.5 \times 10^{16} \mathrm{~cm}^{-3}\right.$; $68700 \mathrm{~cm}^{2} \mathrm{~V}^{-1} \mathrm{~s}^{-1}$ ).

We performed circularly polarized pump-probe experiments using transient induced Faraday rotation, similar to those described elsewhere [4-6], with the difference being

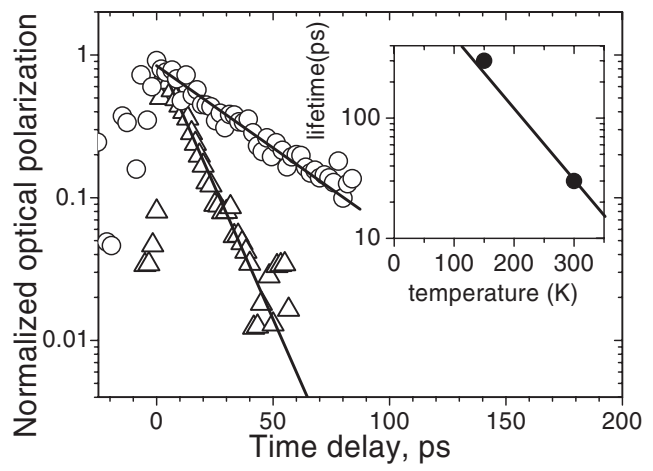

FIG. 1. The optical polarization decay from the bipolar (degenerate) circularly polarized pump-probe experiment at $6 \mu \mathrm{m}$ wavelength and $300 \mathrm{~K}$, for intrinsic (open triangles) and $n$-type doped (open circles) InSb. The solid lines are exponential fits to the data. Inset: spin lifetime determined from the exponential fits for the $n$-type sample (from the present work and from [9]), along with the theoretical prediction from Eq. (1). 
that the pump and the probe originated from two different lasers. The pump beam was the free electron laser, FELIX, which produces (sub-) picosecond pulses and is continuously tunable from 4-250 $\mu \mathrm{m}$. The probe beam was taken from a solid state laser system (pumped by an amplified mode-locked Ti:sapphire laser) producing subpicosecond midinfrared pulses from 3.3-18 $\mu \mathrm{m}$. The lasers were synchronized with an rf phase locked loop. With the chosen sample material, InSb, FELIX can reach both above and below the gap, and we were thus able to investigate easily both interband and intraband excitations without any modification of the experimental apparatus. The sample doping causes a Moss-Burstein shift of the interband absorption edge to wavelength shorter than the room temperature bandgap of InSb (7 $\mu \mathrm{m} \equiv 0.18 \mathrm{eV})$. Therefore the probe laser was set at a wavelength of $6 \mu \mathrm{m}(\equiv 0.20 \mathrm{eV})$ and remained fixed throughout the experiment.

First, the pump laser was set at $6 \mu \mathrm{m}$ wavelength, degenerate with the probe. Both beams were linearly polarized. The pump and probe pulse energies were approximately $1 \mu \mathrm{J}$ and $10 \mathrm{~nJ}$, respectively, and they were focused to spot sizes of approximately $100 \mu \mathrm{m}$. The decay time (data not shown) was $1 \mathrm{~ns}$, and this is the interband recombination time and has been shown previously to be determined by Auger recombination in this material [24].

For the transient Faraday rotation experiment a variable wavelength quarter-wave plate was then inserted into the pump beam. The probe remained linearly polarized (vertically). An uncoated (nonpolarizing) ZnSe beam splitter was placed in the probe beam after the sample and an analyzer was placed in one of the arms (the "signal") at $45^{\circ}$ to the incoming polarization, and the other arm (the "reference") was unpolarized. Matched, cooled $\mathrm{HgCdTe}$ detected the signal and reference simultaneously and the ratio was used as a measure of the induced Faraday rotation, eliminating any change in magnitude of the transmitted probe due to pulse amplitude fluctuations, etc. The decay time of the transient Faraday rotation due to interband bipolar pumping, Fig. 1, gives a spin lifetime of $38 \mathrm{ps}$ for the doped sample ME1722 and 12 ps for the lightly doped sample ME1654.

We then tuned the pump laser to the far infrared at $11 \mu \mathrm{m}(\equiv 0.11 \mathrm{eV})$ and readjusted the variable quarterwave plate in the pump beam for the new wavelength. Nothing else was changed. The new pump pulse energy was $2 \mu \mathrm{J}$ and the spot size was $200 \mu \mathrm{m}$, i.e., the irradiance at the sample was very similar to the degenerate experiment. At this wavelength, well below the band gap of InSb, the strongest absorption process is free-carrier (Drude) absorption of the electrons. It has previously been shown that this process can be used to generate spin polarizations though with a rather weaker selection rule than for interband transitions [25]. Free-carrier absorption is a second order process that involves electron-photon absorption to an intermediate state, followed by phonon scattering. The dominant pathway involves intermediate states within the conduction band, but this is spin independent. However, processes with the intermediate state in the valence band give rise to spin selectivity in exactly the same way as for interband transitions, so that a small fraction of electrons become polarized. In GaAs the number of spins per absorption was estimated to be about $10^{-6}$ for $h \nu=10 \mathrm{meV}$ [25], but in our case this is about 2500 times larger due to the larger spin-orbit coupling \& smaller gap of InSb, and the larger pump photon energy. The probe was left exactly as before, at $6 \mu \mathrm{m}$, and therefore had the same sensitivity to spin polarization in the sample as for the interband pumping experiment. A significantly shorter spin lifetime of 14 ps was measured (solid circles Fig. 2) for the monopolar pump experiment. In comparison, this lifetime is about a third of that obtained with bipolar pumping (open circles Fig. 2).

In order to explain the bipolar experiment, and its relationship with the monopolar result we note that the scattering of minority and majority carriers may be treated the same as ionized impurity scattering, and we may therefore calculate the effect of the photoholes on the orbital electron momentum scattering time using the Brooks-Herring formula [26]

$$
\begin{aligned}
\tau_{p}^{i i}= & \frac{2^{9 / 2} \pi \varepsilon^{2} m^{* 1 / 2} E_{k}^{3 / 2}}{e^{4} N_{I}}\left\{\log \left(1+\frac{8 m^{*} E_{k}}{\hbar q_{0}^{2}}\right)\right. \\
& \left.-\frac{1}{1+\hbar q_{0}^{2} / 8 m^{*} E_{k}}\right\}^{-1},
\end{aligned}
$$

where $m^{*}$ is the electron effective mass, $\varepsilon$ is the dielectric

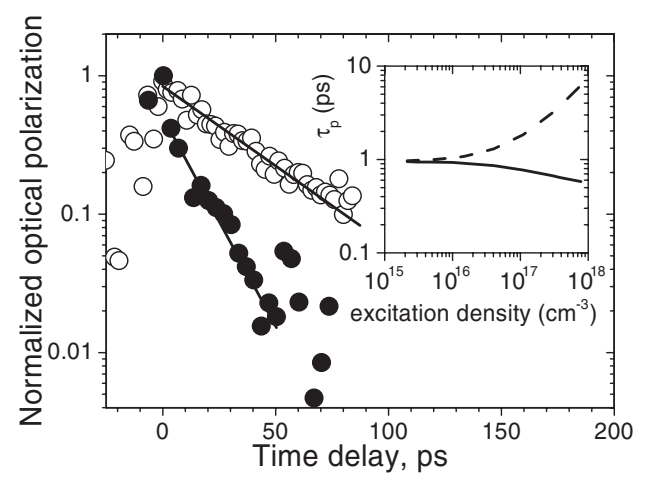

FIG. 2. Comparison of the optical polarization decay for the bipolar and monopolar circularly polarized pump-probe experiments at $300 \mathrm{~K}$. The probe beam was at $6 \mu \mathrm{m}$ wavelength in both cases, and the pump was either at $6 \mu \mathrm{m}$, i.e., degenerate with the probe (open circles) or at $11 \mu \mathrm{m}$ (solid circles). The $11 \mu \mathrm{m}$ pump results in a spin decay that is clearly faster than for $6 \mu \mathrm{m}$ pumping. The solid lines are exponential fits to the data. The inset shows the calculated electron momentum relaxation time due to collisions with ionized impurities only (dashed curve) and with both ionized impurities and photoholes (solid curve), as functions of the photoexcited carrier density. Substitution of $\tau_{p}$ into Eq. (1) gives $\tau_{\mathrm{DP}}$. 
permittivity, $N_{I}$ is the ionized impurity concentration, and the screening length is given by

$$
q_{0}^{2}=\frac{e^{2} m^{*}}{\pi \varepsilon_{0} \varepsilon_{\infty} \hbar^{2}}\left(\frac{3 n_{e}}{\pi}\right)^{1 / 3},
$$

where $n_{e}$ is the total electron concentration. For the total lifetime under bipolar excitation we replace $N_{I}$ by the sum of the donor concentration and the photohole concentration. Results for the momentum scattering time for the two cases (scattering by donors only or by both donors and photoholes) are shown in the inset of Fig. 2. In the monopolar experiment no photocarriers are created, and the momentum scattering time in this limit is $1 \mathrm{ps}$. In the bipolar experiment at bleaching (i.e., when the electron and hole quasi-Fermi energies are separated by the pump photon energy) the photohole concentration is $4 \times 10^{17} \mathrm{~cm}^{-3}$. In this case the momentum scattering time by ionized impurities alone is $4 \mathrm{ps}$, and has risen because the rise in electron energy means that they are less affected by the ion potential. On the other hand, when the photoholes are taken into account, this effectively increases the number of scatterers, so that the total momentum time has fallen to $0.6 \mathrm{ps}$, as shown in Fig. 2. Finally, this means that the $\tau_{\mathrm{DP}} \propto$ $1 /\left(E_{k}^{3} \tau_{p}\right)$ is predicted to increase by a factor of about 2 going from monopolar to bipolar pumping, in reasonable agreement with the experimental results (from 14 to $38 \mathrm{ps}$ ). We have used the bleaching condition again to find the average value $\left\langle E_{k}^{3}\right\rangle=(0.10 \mathrm{eV})^{3}$ in the bipolar experiment, which is very similar to the excitation energy in the monopolar case. The implications for the pure (intrinsic) sample are that the observed bipolar lifetime of $12 \mathrm{ps}$ has also been increased by the photopumping, and that the true DP lifetime is about $6 \mathrm{ps}$. This cannot of course be verified by direct experiment because monopolar pumping is not possible with an intrinsic sample.

Finally, we must consider the effects (if any) of hot electrons on the validity our assumption of motional narrowing. Taking the measured bipolar spin lifetime in the doped sample (38 ps) and the calculated $\tau_{p}(0.6 \mathrm{ps})$, using Eq. (1) we find $\Omega=0.2 \mathrm{ps}^{-1}$, and $\Omega \tau_{p}=0.1$. The value of $\Omega$ is consistent with that found using a reduced value of $\alpha=0.01$ at high $k$ [23], and assuming $Q=0.05$ [7] and $E_{k}=0.10 \mathrm{eV}$. Moving to the monopolar experiment, using $E_{k}=0.11 \mathrm{eV}$ and $\tau_{p}=1 \mathrm{ps}$, we find that $\Omega \tau_{p}=$ 0.3. Thus, although the electrons are hot, in both cases $\Omega \tau_{p} \ll 1$ and, consequently, the motional narrowing condition applies. Finally we note that $\left\langle E_{k}^{3}\right\rangle$ is very similar in both cases, so the different lifetime is not due to different electron distributions. We may therefore assume that differences between the two results are due to the effects of holes on $\tau_{p}$.

In conclusion, we have demonstrated clearly that through the presence of the photoholes there is a difference between monopolar and bipolar pumping of a bulk semiconductor. The spin lifetime in the monopolar regime was shown to decrease by about a factor of between 2 and 3 compared to the bipolar pumping case. This could have important implications for the physics of future spintronic devices. It is suggested that the explanation for this shortening of the lifetime for monopolar pumping is that the photoholes lower the electron mobility, which for the DP mechanism lengthens the spin relaxation time. In the case of the EY or BAP mechanism the injection of holes and lowering of the mobility would have the opposite effect.

We gratefully acknowledge support by EPSRC-UK (GR/R74178, EP/C511999), FOM-NL, and the European Community (G5RD-CT-2001-00535 and I3 IA-SFS).

* Present address: Department of Physics and Astronomy, University of Sheffield, Sheffield S3 7RH, United Kingdom.

[1] S. Datta and B. Das, Appl. Phys. Lett. 56, 665 (1990).

[2] R. Fiederling et al., Nature (London) 402, 787 (1999).

[3] Y. Ohno et al., Nature (London) 402, 790 (1999).

[4] Y. Kato, R.C. Myers, A.C. Gossard, and D.D. Awschalom, Nature (London) 427, 50 (2004).

[5] O.Z. Karimov et al., Phys. Rev. Lett. 91, 246601 (2003).

[6] M. A. Brand et al., Phys. Rev. Lett. 89, 236601 (2002).

[7] G.E. Pikus and A.N. Titkov, in Optical Orientation, edited by F. Meier and B. Zakharchenya (North-Holland, New York, 1984), p. 84.

[8] A. Tackeuchi, T. Kuroda, S. Muto, and O. Wada, Physica B (Amsterdam) 272, 318 (1999).

[9] P. Murzyn et al., Phys. Rev. B 67, 235202 (2003).

[10] T. F. Boggess et al., Appl. Phys. Lett. 77, 1333 (2000).

[11] P. Murzyn et al., Appl. Phys. Lett. 83, 5220 (2003).

[12] B. N. Murdin et al. (to be published).

[13] K. C. Hall et al., Phys. Rev. B 68, 115311 (2003).

[14] S. D. Ganichev et al., Phys. Rev. Lett. 88, 057401 (2002).

[15] W. Zawadski and P. Pfeffer, Semicond. Sci. Technol. 19 R1 (2004).

[16] T. Koga, J. Nitta, T. Akazaki, and H. Takayanagi, Phys. Rev. Lett. 89, 046801 (2002).

[17] S. D. Ganichev et al., Phys. Rev. Lett. 92, 256601 (2004).

[18] P. R. Hammar and M. Johnson, Phys. Rev. Lett. 88, 066806 (2002).

[19] M. I. D'yakonov and V. I. Perel, Sov. Phys. JETP 33, 1053 (1971); Sov. Phys. Solid State 13, 3023 (1972).

[20] R. J. Elliott, Phys. Rev. 96, 266 (1954); Y. Yafet, in Solid State Physics, edited by F. Seitz and D. Turnbull (Academic, New York, 1963), Vol. 14.

[21] G. L. Bir, A. G. Aronov, and G. E. Pikus, Sov. Phys. JETP 42, 705 (1975).

[22] W. H. Lau, J. T. Olesburg, and M. E. Flatte, Phys. Rev. B 64, 161301 (2001).

[23] M. Cardona, N. E. Christensen, and G. Fasol, Phys. Rev. B 38, 1806 (1988).

[24] B. N. Murdin et al., Inst. Phys. Conf. Ser. 144, 267 (1995).

[25] S. A. Tarasenko et al., J. Supercond. 16, 419 (2003).

[26] B. K. Ridley, Quantum Processes in Semiconductors (Oxford University, New York, 1993), p. 176. 